\title{
ABOUT THE POSSIBILITY OF CREATING AN EFFICIENT ENERGY ANALYZER OF CHARGED PARTICLE BEAMS BASED ON AXIALLY- SYMMETRIC OCTUPOLE-CYLINDRICAL FIELD
}

\author{
Kambarova Zh.T. ${ }^{1 *}$, Saulebekov A.O. ${ }^{2}$, Kopbalina K.B. ${ }^{3}$, Tussupbekova A.K. ${ }^{1}$, \\ Saulebekova D.A. ${ }^{4}$
}

\author{
1 E.A. Buketov Karaganda University, Karaganda, Kazakhstan, kambarova@bk.ru \\ ${ }^{2}$ Lomonosov Moscow State University, Kazakhstan branch, Nur-Sultan, Kazakhstan \\ ${ }^{3}$ Karaganda Technical University, Karaganda, Kazakhstan \\ ${ }^{4}$ Institute Curie, Sorbonne University, Paris, France
}

\begin{abstract}
One of the problems in creating systems for energy analysis of charged particles beams is to determine the deflecting field and calculation the shape of the deflecting electrodes. This article is devoted to the study of the possibility of creating an effective energy analyzer of charged particle beams based on multipole electrode systems. A previously unstudied type of a multipole-cylindrical field - an electrostatic axially-symmetrical octupole-cylindrical field was chosen as the deflecting field. The field is formed by using the superposition of an electrostatic cylindrical field and a circular octupole of various contributions. The family of the equipotentials of cylindrical octupoles with planes of symmetry and antisymmetry is calculated. The calculation and analysis of equipotential portraits of the electrostatic axially-symmetric octupole-cylindrical fields with different weight contributions of the cylindrical field and circular octupole are carried out.
\end{abstract}

Keywords: energy analyzer, deflecting field, octupole-cylindrical field, equipotential, equipotential portraits, multipole electrode systems.

\section{Introduction}

One of the ways to improve the work of the instrumentation of energy analysis of charged particle beams is to modify the deflecting field by changing the shape of the outer electrode of a cylindrical mirror. A cylindrical mirror has a number of advantages, such as high energy resolution, simplicity of construction, etc. Its work is based on the focusing and dispersing action of the field in the space between two coaxial cylindrical electrodes on a charged particlesbeam. The theory and the possibility of practical application of a cylindrical mirror were studied in detail by a group of scientists led by Professor V.V. Zashkvara [1].

The cylindrical mirror type energy analyzers have found wide application in the study of resonance phenomena in gases, in spectroscopy for chemical analysis, for obtaining spectra of secondary electrons, photoelectrons, field electrons, Auger electrons, as well as in space research, in studying the interaction of atomic particles with the solid surface and plasma diagnostics. The cylindrical mirror analyzer has become the basic element of electron spectrometers of various purposes, manufactured in the countries of near and far abroad by leading instrument-making firms [2].

The new class of potential fields called multipole-cylindrical fields is first proposed and classified by V.V. Zashkvara and N.N. Tyndyk in [3-6]. A multipole-cylindrical field is formed by using a superposition of an electrostatic cylindrical field and a circular multipole of various contributions.

The purposes of this work are to study the possibility of creating an effective mirror energy analyzer of charged particle beams based on an axially-symmetric electrostatic octupole-cylindrical field, to calculate and construct equipotential portraits of the corresponding fields for various contributions of the cylindrical field and circular octupole, and to analyze the obtained field equipotentials. Consideration and analysis of superpositions of these fields will make it possible to predict the prospects of choosing one or another scheme of energy analyzer for space experiments. 


\section{Calculation and analysis}

An electrostatic multipole-cylindrical field is formed in the space between two axially symmetric coaxial electrodes, the inner (2) of which has a cylindrical shape ( $r_{0}$ radius) and is at zero potential; to the outer curvilinear electrode (3) is supplied deflecting potential. The shape of the outer deflecting electrode is interested. The profile of the outer deflecting electrode (3) (Fig. 1) coincides with one of the equipotentials of the cylindrical multipole. A charged particles beam can be introduced into the area of the mirror field and removed from it through the corresponding aperture windows in the cylindrical electrode.

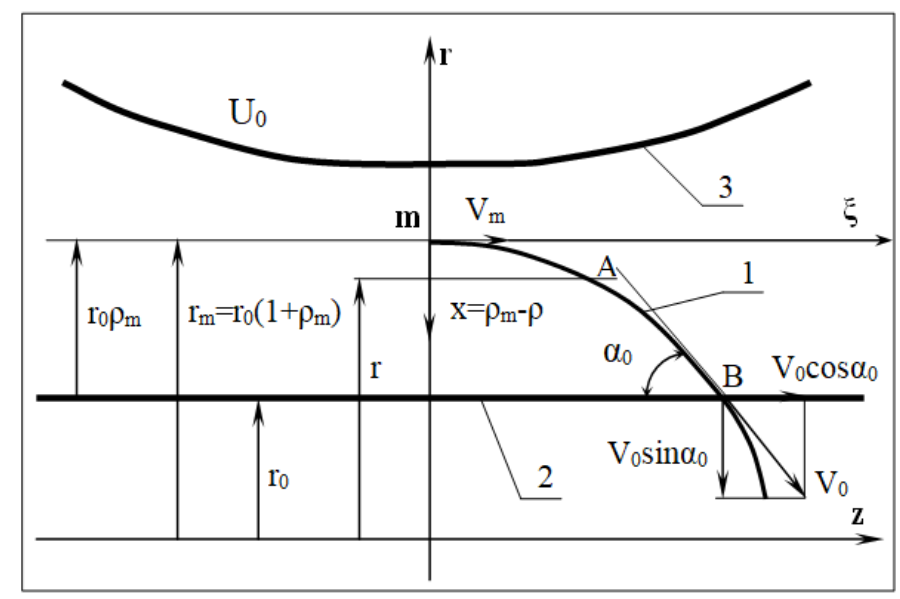

Fig.1. The electron-optical scheme of an energy analyzer based on a multipole-cylindrical field ${ }^{\wedge}$

1- Trajectory of charged particles, 2 - cylindrical electrode, 3 - outer deflection electrode

Earlier, the corpuscular-optical schemes of mirror energy analyzers of charged particle beams based on electrostatic multipole-cylindrical fields were studied in sufficient detail; a large number of works, for example, [7-11], were aimed at studying their corpuscular-optical characteristics, at finding optimal schemes with high quality focusing and energy resolution. Using the electrode system of an axially-symmetric electrostatic decapole-cylindrical field to build energy analyzer of charged particles for space research was proposed in [12]. It was determined from the calculations that the value of the specific dispersion in energy, which characterizes the energy resolution of the decapole-cylindrical field, calculated for particles with an initial angular divergence of $12^{\circ}$, is twice the value of the specific dispersion of the classical cylindrical mirror analyzer. The device makes it possible to register elementary particle beams from outer space, to obtain various spectra with high resolution.

According to [4], the $g(R, \xi)$ field of energy analyzer can be designed as a superposition of the base field and a set of circular multipoles which coaxial with the base field. In the case of an electrostatic deflection field, this design looks like this:

$$
g(R, \xi)=\ln R+q U_{q}(R, \xi)+s U_{S}(R, \xi)+\omega U_{o c t}(R, \xi)+d U_{d e c}(R, \xi)+\ldots
$$

where $\ln R$ is the basic cylindrical field, $U_{q}, U_{S}, U_{o c t}, U_{d e c}$ are circular multipoles (quadrupole, hexapole, octupole, decapole, etc., $q, s, \omega, d, \ldots$ are the weight coefficients of the multipoles, $R=r / r_{0}$ and $\xi=z / z_{0}$ are the relative radial and axial coordinates, $r_{0}$ is the radius of the axially circular trajectory of the analyzed beam of charged particles (Fig. 1).

The uniqueness of the multipole-cylindrical field (5) is that circular multipoles have different effects on the electron-optical parameters of the energy analyzer of various orders:

- the quadrupole component in (5) determines the first-order angular focusing conditions - linear energy dispersion, magnification;

- the connection of the hexapole component affects (along with the quadrupole) the second-order angular aberrations; 
- the connection of the octupole affects the characteristics, starting from the third-order, etc.

The potential of an octupole-cylindrical field is described in the $r, z$ coordinate system by the following expression:

$$
U(r, z)=\mu \ln r+\omega U_{o c t}(r, z)
$$

where $\ln r$ is the potential of the cylindrical field, $\mu$ is the coefficient specifying the weight contribution of the cylindrical field $\ln r, U_{\text {oct }}$ is the potential of a first type circular octupole, $\omega$ is the weight contribution of the circular octupole.

The $U_{\text {oct }}(\rho, \xi)$ functions constructed according to the summation symmetry rule are below:

$$
\begin{aligned}
& U_{o c t}(\rho, \xi)=\frac{1}{4 !} \xi^{4}+\frac{1}{2} \xi^{2}\left\{\frac{1}{4}\left[1-(1+\rho)^{2}\right]+\frac{1}{2} \ln (1+\rho)\right\}+ \\
& +\frac{1}{64}(1+\rho)^{4}+\frac{1}{16}(1+\rho)^{2}-\frac{1}{8} \ln (1+\rho)\left[\frac{1}{2}+(1+\rho)^{2}\right]-\frac{5}{64} \\
& U_{o c t}^{\prime}(\rho, \xi)=\frac{1}{5 !} \xi^{5}+\frac{1}{3 !} \xi^{3}\left\{\frac{1}{4}\left[1-(1+\rho)^{2}\right]+\frac{1}{2} \ln (1+\rho)\right\}+ \\
& +\frac{1}{64} \xi\left\{(1+\rho)^{4}+4(1+\rho)^{2}-8 \ln (1+\rho)\left[\frac{1}{2}+(1+\rho)^{2}\right]-5\right\}
\end{aligned}
$$

Field (7) is called a component of a cylindrical circular octupole with a symmetry plane. For this field $U_{o c t}(\rho,-\xi)=U_{o c t}(\rho, \xi)$. Field (8) is called a component of a cylindrical circular octupole with an antisymmetry plane. This field satisfies the requirement for antisymmetry relative to the plane $\xi=0$ : $U_{o c t}^{\prime}(\rho,-\xi)=-U_{o c t}^{\prime}(\rho, \xi)$.

After expanding (7) and (8) into series in $\rho$ and $\xi$ around the axial circle $\rho=0, \xi=0$, and selecting the group of terms with the least powers, we get:

$$
\begin{aligned}
& U_{\text {oct }}(\rho, \xi) \sim \quad{ }^{2} \rho^{2}+\rho^{4} \quad, \\
& \left.U_{\text {oct }}^{\prime}(\rho, \xi) \sim{ }_{\curlywedge}^{\prime}-2 \xi^{2} \rho^{2}+\rho^{4}\right)
\end{aligned}
$$

Function (9) is an analogue of a planar multipole with a rectilinear axis in a symmetric representation an octupole, and function (10) is a component of a planar multipole with a rectilinear axis, but in an antisymmetric representation.

A family of equipotentials of an electrostatic axially-symmetric octupole-cylindrical field is calculated. Fig. 2 shows field equipotentials $U_{\text {oct }}$ and $U_{o c t}^{\prime}$ at intervals of 0.1 of the relative value of the potential. In the $\rho, \xi$ plane section, each of the fields is divided into alternating regions in which the potentials are opposite to the sign. These areas are separated by lines of zero potential, close to straight lines and converging at the nodal point $O_{1}(\rho=\xi=0)$. From Fig. 2 it follows that for each type of symmetry relative to the plane $\xi=0$, the presented fields are formed by octupole sets of electrodes carrying an alternating potential.

Let's get an presentation of the structure of the superposition of the electrostatic octupole and the cylindrical field. When the fields are added, the central circle of the octupole isaligned with the zero equipotential of the logarithmic field. Series of Fig. 3 show the families of equipotentials of the superposition of a cylindrical field and a cylindrical octupole:

$$
U(\rho, \xi)=\omega U_{o c t}(\rho, \xi)+\mu \ln (1+\rho)
$$




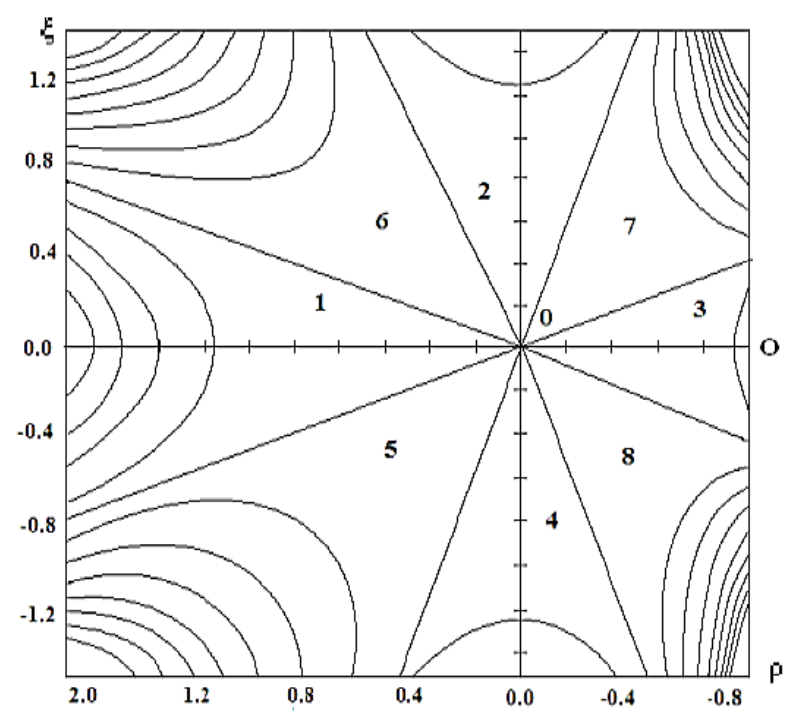

$a$

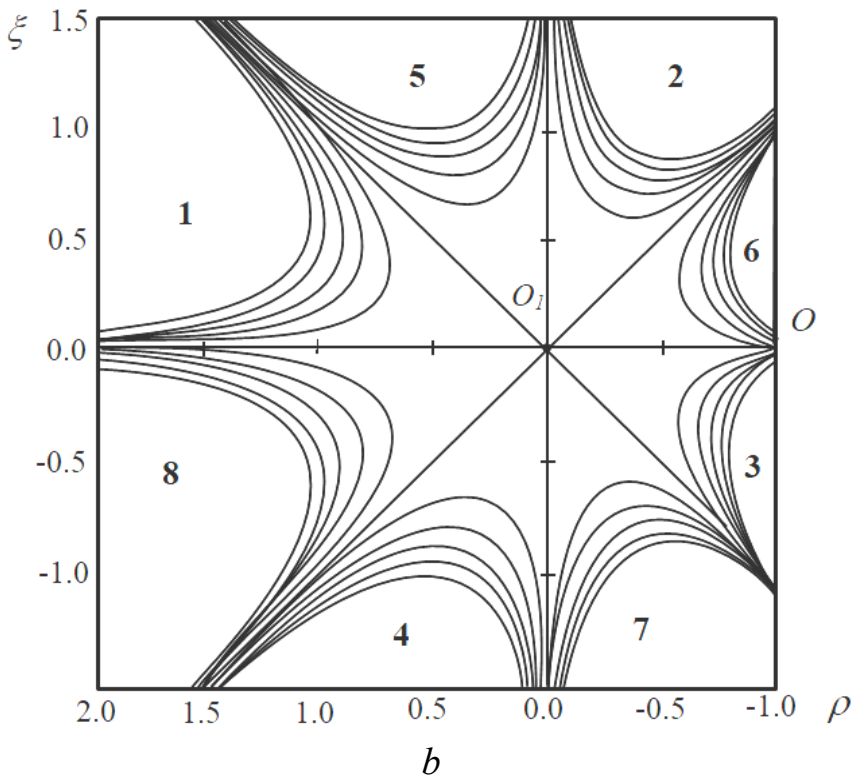

Fig. 2. Family of equipotentials of a cylindrical octupole having a symmetry plane $(a)$ and an antisymmetry plane $(b)$

For a series of Fig.3 (a-f) $\omega=-1.5,-1,0.001,0.005,1,1.5$ at a constant value of the contribution of the cylindrical field $\mu=1$. From the figures we can trace how gradually with increasing $\omega$ the weight contribution of the circular octupole the structure of the field changes. At small values of $\omega$ (Fig. $3 c$ and $d$ ), the structure of the field is transformed from an octupole to a field close to cylindrical, and the nodal point $O_{1}$ is also eliminated. For $\omega<0$ (Fig. $3 a$ and $b$ ), the removal of vertices occurs along the $\rho$ axis, which then leads to the displacement of regions 1 and 3 (Fig. 2a).

Series of Fig. $4(a-d)$ show the families of equipotentials of the superposition of a cylindrical field and a cylindrical octupole. For this series of Fig. $4(a-f)$ the values of the weight contribution of the cylindrical field $\mu=2,5$; 1 at a constant value of the weight contribution of the circular octupole $\omega=1$. As can be seen from the figures, the addition of a cylindrical field $\ln (1+\rho)$ to the circular octupole $U_{\text {oct }}(\rho, \xi)$ leads to a change in the field, namely, the approach of regions 2 and 4 to the nodal point. It was determined from the analysis of equipotential portraits of $U(\rho, \xi)=\omega U_{o c t}(\rho, \xi)+\mu \ln (1+\rho)$ field, that with a small change in the value of the weight contribution $\mu$ of the cylindrical field does not lead to large transformations of the field. 


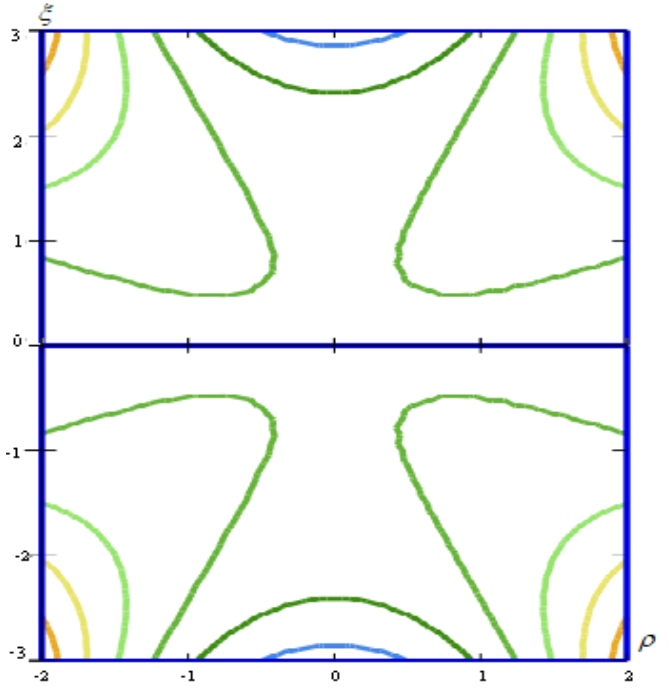

a)

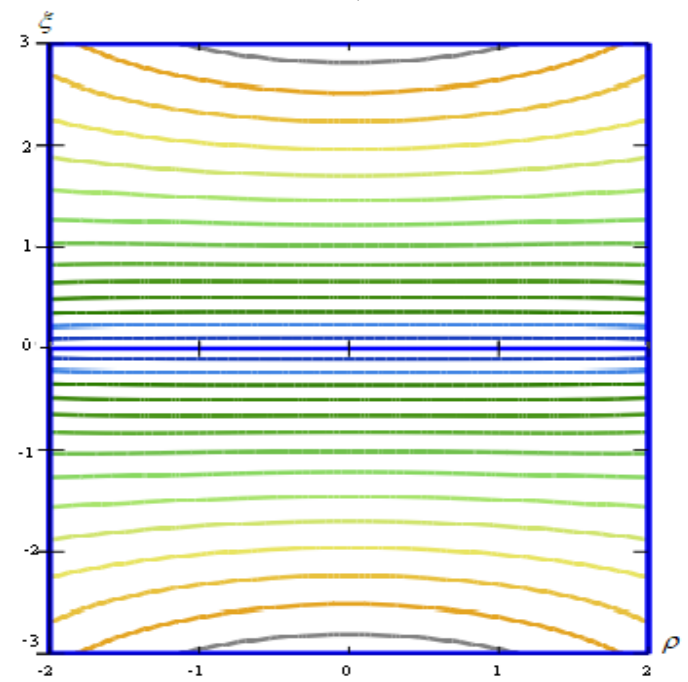

c)

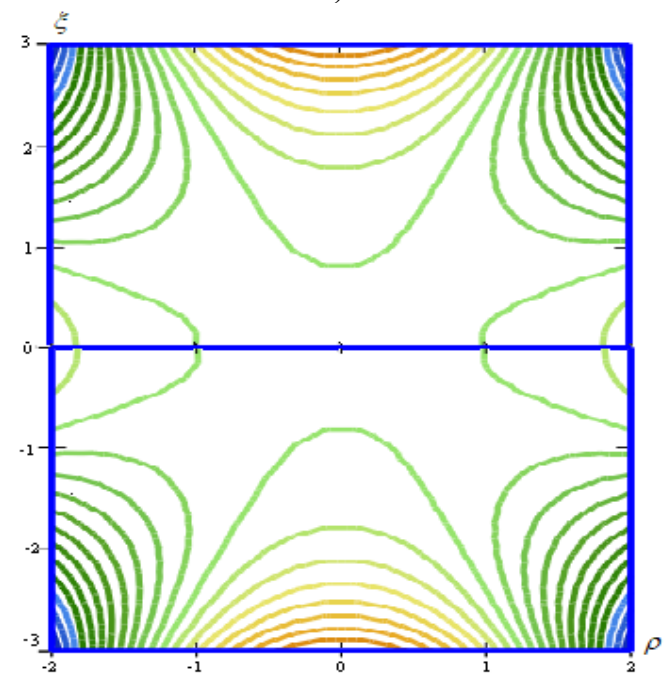

e)

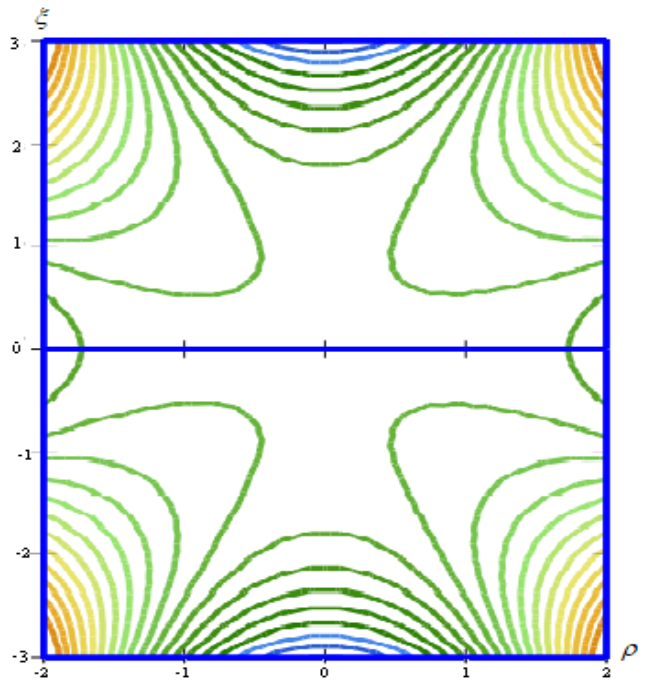

b)

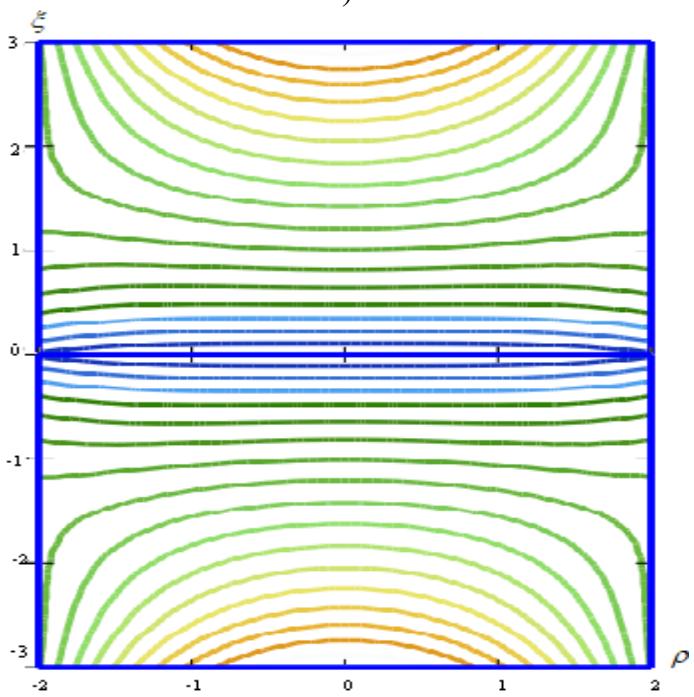

d)

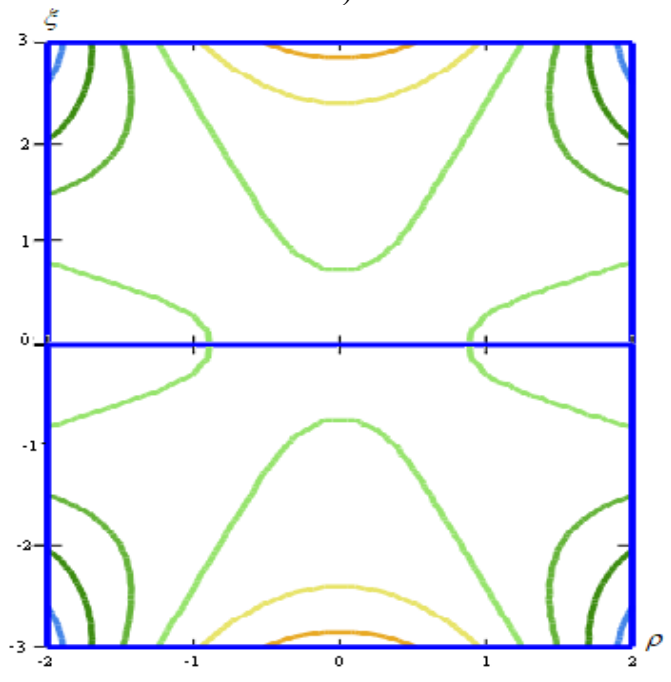

f)

Fig. 3. Equipotential fields $U(\rho, \xi)=\omega U_{\text {oct }}(\rho, \xi)+\mu \ln (1+\rho)$ at $\rho(-2,2)$ :

a) $\omega=-1.5, \mu=1$; b) $\omega=-1, \mu=1$; c) $\omega=0.001, \mu=1$

d) $\omega=0.005, \mu=1$; e) $\omega=1, \mu=1$; f) $\omega=1.5, \mu=1$. 


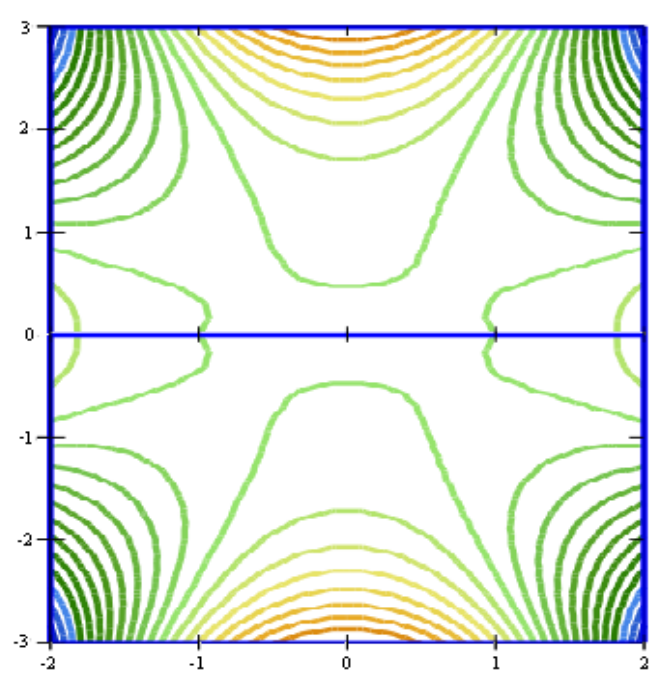

a)

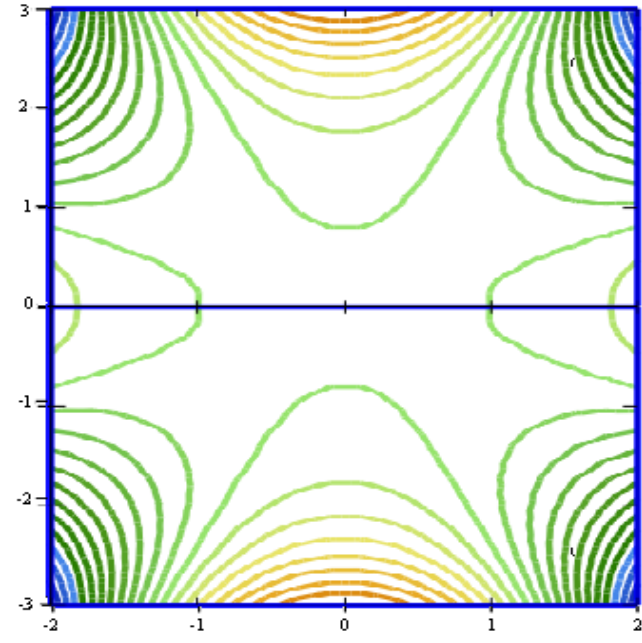

c)

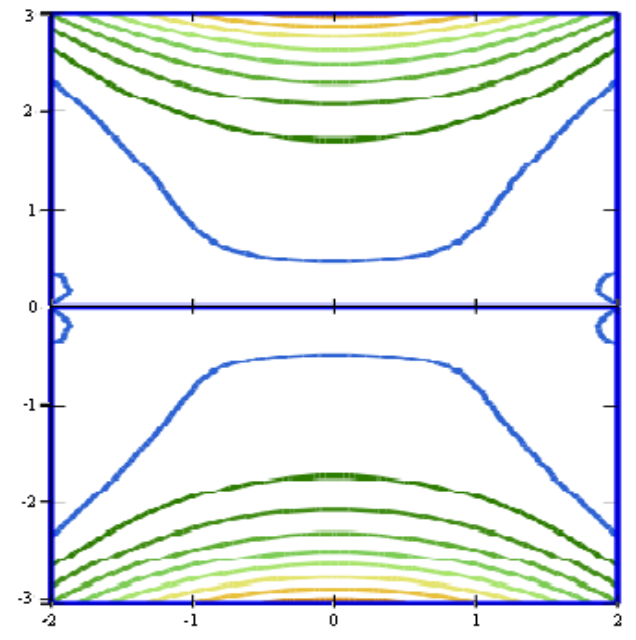

b)

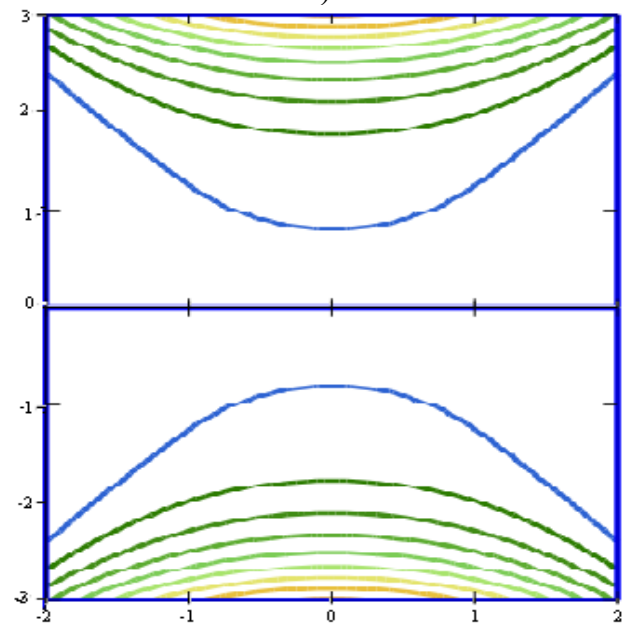

d)

Fig.4. Equipotential fields $U(\rho, \xi)=\omega U_{o c t}(\rho, \xi)+\mu \ln (1+\rho)$ :

a) $\mu=2.5, \omega=1 ; \rho(-2,2)$ : b) $\mu=2.5, \omega=1 ; \rho(-1,1)$

c) $\mu=1, \omega=1 ; \rho(-2,2)$ d); $\mu=1, \omega=1 ; \rho(-1,1)$

Figure 5 shows an example of the image of the equipotential field $U(\rho, \xi)=\omega U_{\text {oct }}(\rho, \xi)+\mu \ln (1+\rho)$ in the three-dimensional coordinate system Surface Plot with the values $\omega=-1.5, \mu=1$.

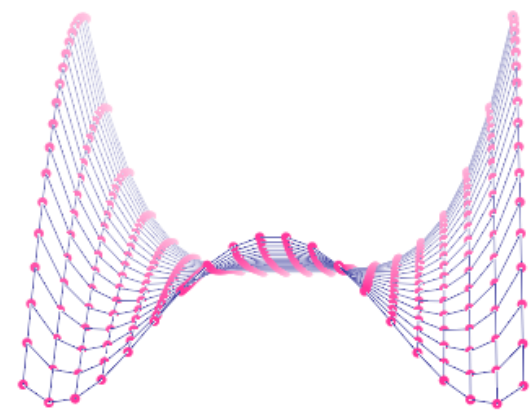

Fig.5. Equipotential field $U(\rho, \xi)=\omega U_{o c t}(\rho, \xi)+\mu \ln (1+\rho)$

in the three-dimensional coordinate system Surface Plot at $\omega=-1.5, \mu=1$ 


\title{
Conclusions
}

The superposition of a cylindrical field and a cylindrical octupole is interesting for the implementation of an axially-symmetric electrostatic mirror consisting of a cylindrical electrode at zero potential and an axially-symmetric deflecting electrode, the generatrix of which coincides with one of the equipotentialsof $U(\rho, \xi)=\omega U_{o c t}(\rho, \xi)+\mu \ln (1+\rho)$ field. A charged particles beam can be introduced into the area of the mirror field and removed from it through the corresponding aperture windows in the cylindrical electrode. Thus, the profile of the outer deflecting electrode is determined from the calculation of the equipotential lines of the octupole-cylindrical field.

\author{
Acknowledgements \\ The research was supported by the Ministry of Education and Science of the Republic of Kazakhstan, \\ Grant number AP09058188
}

\section{REFERENCES}

1 Zashkvara V.V., Korsunsky M.I., Kosmachev O.S. Focusing properties of an electrostatic mirror with a cylindrical field. Zhurnal tekhnicheskoy fiziki, 1966, Vol. 36, pp. 132 - 138. [in Russian].

2 Zashkvara V.V., Ashimbaeva B.U., Bylinkin A.F. Spectrograph regime in an energy analyzer of two cylindrical mirrors. Zhurnal tekhnicheskoy fiziki, 1988, Vol.58, No. 10. - pp.2021-2025.[in Russian]

3 Zashkvara V.V., Tyndyk N.N. Axial symmetric electrostatic multipoles and their applications.Zhurnal tekhnicheskoy fiziki,1991, Vol.61, No. 4. - pp.148-157.[in Russian]

4 ZashkvaraV.V., Tyndyk N.N. Electrostatic axially symmetric multiple in deflector-type analyzers. Nuclear Instruments \& Methods in Physics Research, 1992, Vol. A313.- pp. 315-327.

5 Zashkvara V.V., Ashimbaeva B.U., Chokin K.Sh. Calculation of trajectories in a multipole cylindrical field. Journal of Electron Spectroscopy and Related Phenomena, 2002, Vol. 122, - pp.195-202.

6 Zashkvara V.V., Tyndyk N.N. The method for the calculation of multipole-cylindrical fields. Nuclear Instruments \& Methods in Physics Research. Section A, 1999, A370, - pp. 223-231.

7 Saulebekov A.O., Vénos D., Kambarova Zh.T., Saulebekova D.A., Vassilyeva K.I., Seidualy K.B. Development of energy analyzer of charged particles based on the basis non-uniform electrostatic field. Eurasian Physical Technical Journal, 2019, Vol.16, No.1(31). pp. 24 - 29.

8 Kambarova Zh.T., Saulebekov A.O. Development of mirror energy analyzer based on electrostatic quadrupole-cylindrical field. Eurasian Physical Technical Journal, 2017, V.14, No. 2 (28). pp. 42 - 47.

9 Assylbekova S.N., Saulebekov A.O., Kambarova Zh.T. Numerical modeling of the electrostatic energy analyzer based on hexapole-cylindrical field with a concave outer electrode. Eurasian Physical Technical Journal, 2015, V.12, No. 1 (23). - pp.25-29.

10 Kambarova Zh.T., Trubitsyn A.A., Saulebekov A.O. Axially symmetric energy analyzer based on the electrostatic decapole-cylindrical field. Technical Physics, 2018, Vol. 63, No. 11, pp. 1667-1671.

11 Kambarova Z.T., Saulebekov A.O., Saulebekova D.A. Modeling of electrostatic decapole-cylindrical mirror analyzer. IOP Conference Series: Materials Science and Engineering, 2017, No. 12, pp. 012078. DOI:10.1088/1757$899 \mathrm{X} / 168 / 1 / 012078$

12 Saulebekov A.O., Venos D., Kambarova $\mathrm{Zh.T}$. The high resolution electrostatic energy analyzer for space research. Eurasian Physical Technical Journal, 2020, V.17(33). pp.163-168. 